\title{
Activation of ERK and P38 by the Addition of Arsenic Trioxide in Flt3-ITD Cells
}

\author{
Sawami Suzuki $^{1}$, Hiroko Inaba ${ }^{2}$, Takashi Satoh ${ }^{1,2}$, Toshio Okazaki ${ }^{1,2}$, Shinichiro Takahashi ${ }^{1,2^{*}}$ \\ ${ }^{1}$ Division of Molecular Hematology, Kitasato University Graduate School of Medical Sciences, Sagamihara, Japan; ${ }^{2}$ Division of \\ Hematology, Kitasato University School of Allied Health Sciences, Sagamihara, Japan. \\ Email: *shin@kitasato-u.ac.jp
}

Received September $20^{\text {th }}, 2011$; revised October $29^{\text {th }}$, 2011; accepted November $14^{\text {th }}, 2011$.

\begin{abstract}
Flt3-internal tandem duplications (Flt3-ITD) is a prevalent mutation in acute myeloid leukemia (AML). We recently reported arsenic trioxide (ATO) and Flt3 inhibition synergize to induce apoptosis in Flt3-ITD cells. However, the signaling effect of ATO in these cells has not been elucidated. Here, we demonstrate that the treatment of ATO potently induces the activation of extracellular regulated kinase (ERK)-mitogen activated protein kinase (MAPK), and modestly activates p38-MAPK in BaF3-Flt3-ITD cells, among other major (PI3-kinase-Akt, c-jun N-terminal kinase [JNK]) signaling pathways examined. In contrast, in BaF3-Flt3-wild type (WT) cells, slight activation of p38, but none for others, was observed. As MAPK kinase (MEK), as well as p38 inhibition is reported to enhance ATO-induced apoptosis in AML and various hematological malignancies, our results suggest that Flt3 mutation status is important for the effect of these combinations.
\end{abstract}

Keywords: Flt3-ITD, ERK, Arsenic Trioxide

\section{Introduction}

Flt3 is a member of the class III receptor tyrosine kinase family, and approximately one third of AML patients have mutation of this gene. The majority of such mutations are ITD in the juxtamembrane domain of Flt3, specifically found in AML, which result in ligand-independent dimerization and tyrosine phosphorylation of the receptor [1,2]. This causes constitutive activation of downstream signaling pathways, including the Ras/MEK/MAPK pathway, leading to aberrant cell growth and differentiation block in leukemia cells [1]. The MEK/MAPK pathway is an important signaling cascade involved in the control of hematopoietic cell proliferation and differentiation [3]. The downmodulation of MEK phosphorylation inhibits proliferation and induces apoptosis of primary AML blasts [4]. ATO has currently widely used in the treatment of the patients with relapsed or refractory APL [5]. It was reported that downmodulation of MEK/ERK phosphorylation significantly enhanced ATO-induced apoptosis in primary APL blasts [6]. Additionally, it was reported that not only in APL blasts, but in AML cases, the combination of ATO with an MEK inhibitor is very efficient $[7,8]$. We recently reported that ATO and Flt3 inhibition synergize to induce apoptosis in Flt3-ITD cells [8]. In this study, we examined the signaling effect of ATO on Flt3-WT and Flt3 mutated cells, to clarify the mechanisms of the specific effect of ATO on Flt3-ITD cells.

\section{Materials and Methods}

\subsection{Cell Culture}

BaF3-Flt3-ITD and BaF3-Flt3-WT cells were recently established in our laboratory [9]. All experiments using BaF3-Flt3-ITD cells were performed under IL-3 deprivation because the Flt3-ITD signal is redundant in the presence of IL-3 stimulation of the cells [10].

\subsection{Westernblot}

For assay of phosphorylated proteins, $3 \times 10^{6}$ of BaF3Flt3-WT and BaF3-Flt3-ITD cells [9] were washed twice with PBS and resuspended with serum free RPMI and seeded into $10 \mathrm{~cm}$ dishes. Then the cells were exposed with FL $(100 \mathrm{ng} / \mathrm{ml})$ in the presence or absence of ATO $(4 \mu \mathrm{M})$. The cells were extracted at indicated time points. Phosphorylated proteins of ERK, p38, JNK and Akt were determined by Westernblot as previously described [11]. 


\section{Results and Discussion}

To uncover the signaling difference between Flt3-ITD and Flt3-WT, we employed BaF3-Flt3-ITD and -WT cells those we established in our laboratory [9]. As shown in Figure 1, in BaF3-Flt3-ITD cells, ERK was potently phosphorylated $30 \mathrm{~min}$ after the addition of 4 $\mu \mathrm{M}$ of ATO and its activation was decreased thereafter. There was a modest effect for the phosphorylation of p38-MAPK in BaF3-Flt3-ITD cells. In contrast, in BaF3-Flt3-WT cells, slight activation of p38-MAPK, but none for others, was observed by the addition of ATO.

It was reported that ATO at clinically achievable concentrations $(2-7 \mu \mathrm{mol} / \mathrm{l})$ activated p38-MAPK in several leukemia cell lines or myeloma cells $[12,13]$. p38-MAPK has been shown to mediate both proapototic/growth inhibitory and antiapoptotic/pro-growth signals in different systems, apparently depending on the stimulus and cell type involved [5,12,14]. Combination of ATO with p38-MAPK inhibition significantly increased the apoptosis and/or growth inhibition induced by ATO treatment in these cells $[12,13]$. Therefore, current results indicate that combination therapy of p38-MAPK inhibitors with ATO might be effective for both Flt3-ITD and WT cells.

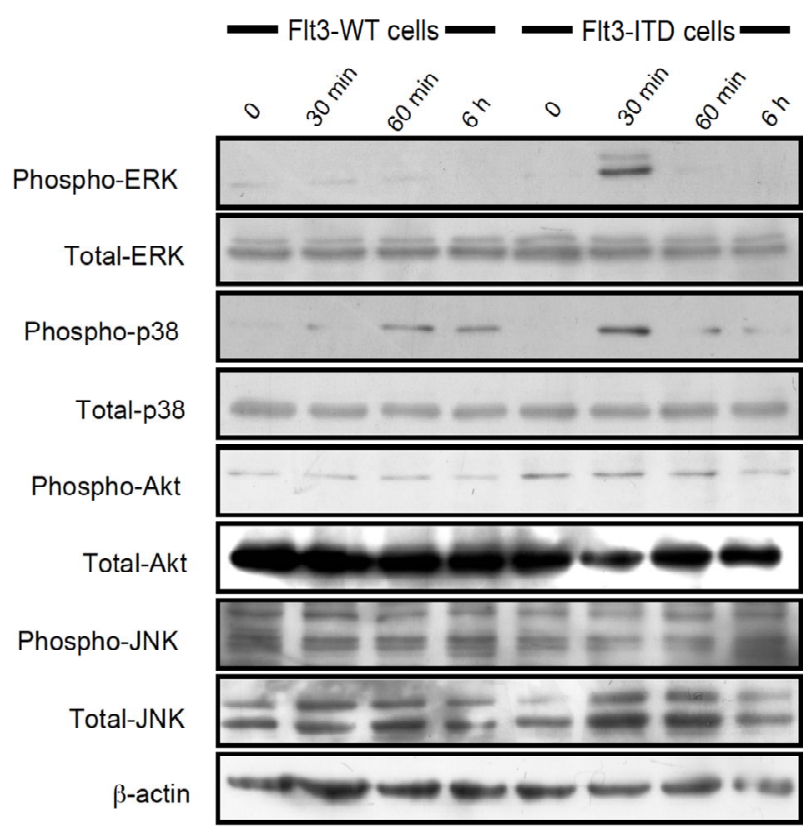

Figure 1. The signaling effect of ATO in BaF3-Flt3-WT and BaF3-FIt3-ITD cells. $3 \times 10^{6}$ of BaF3-FIt3-WT and BaF3Flt3-ITD cells were washed twice with PBS and resuspended with serum free RPMI and seeded into $10 \mathrm{~cm}$ dishes. Then the cells were exposed with FL $(100 \mathrm{ng} / \mathrm{ml})$ in the presence or absence of ATO $(4 \mu \mathrm{M})$. The cells were extracted at indicated time points. Phosphorylated proteins of ERK, p38, Akt and JNK were determined. $\beta$-actin served as a loading control. The results presented are representative of two to three different experiments.
Common chemotherapeutic drugs usually induce a wide range of cytotoxic effects on hematopoietic stem cells or progenitor cells of other tissues. In addition, there are many serious side effects of chemotherapy. In contrast, the therapeutic dose of ATO is associated with an acceptable toxicity level without bone marrow hypoplasia or alopecia [15]. Although QT interval prolongation APL differentiation syndrome are the most serious complications observed in patients with ATO, ATO is well tolerated and toxicities are manageable and reversible. From these points of view, combination therapy with ATO may be advantageous in leukemia therapy. It was noted that ATO is a potent stimulator of ERK and AP-1 transactivational activity and an efficient inducer of c-fos and c-jun gene expression [16,17]. Induction of c-jun and c-fos by ATO is also associated with the activation of JNK [16]. However, a subsequent study indicated that at a therapeutic dose for AML $(<5 \mu \mathrm{M})$, ATO dominantly induces ERK, but not JNK phosphorylation [18], which is consistent with the current result of our study. As MEK inhibitors are promising agents for the treatment of AML [19-22], our results provide an evidence that the status of Flt3 receptor is responsible for the effect of MEK inhibition in combination with ATO.

\section{Acknowledgements}

This work was supported in part by Grants-in-Aid for Scientific Research (No. 23590687) from the Ministry of Education, Science and Culture, Japan, Takeda Science Foundation, and a foundation from Kitasato University School of Allied Health Sciences (Grant-in-Aid for Research Project, No. 2010-1004, No. 2011-1001). There are no conflicts of interest.

\section{REFERENCES}

[1] S. Takahashi, "Downstream Molecular Pathways of Flt3 in the Pathogenesis of Acute Myeloid Leukemia: Biology and Therapeutic Implications,” Journal of Hematology \& Oncology, Vol. 4, No. 13, 2011, pp. 1-10. doi:10.1186/1756-8722-4-13

[2] S. Takahashi, "Current Findings for Recurring Mutations in Acute Myeloid Leukemia,” Journal of Hematology \& Oncology, Vol. 4, No. 36, 2011, pp. 1-11. doi:10.1186/1756-8722-4-36

[3] M. B. Miranda, T. F. McGuire and D. E. Johnson, "Importance of MEK-1/-2 Signaling in Monocytic and Granulocytic Differentiation of Myeloid Cell Lines,” Leukemia, Vol. 16, No. 4, 2002, pp. 683-692. doi:10.1038/sj.leu.2402400

[4] M. Milella, S. M. Kornblau, Z. Estrov, et al., "Therapeutic Targeting of the MEK/MAPK Signal Transduction Module in Acute Myeloid Leukemia," Journal of Clinical Investigation, Vol. 108, No. 6, 2001, pp. 851-859. doi:10.1172/JCI12807

[5] S. Takahashi, "Combination Therapy with Arsenic Tri- 
oxide for Hematological Malignancies," Anti-Cancer Agents in Medicinal Chemistry, Vol. 10, No. 6, 2010, pp. 504-510.

[6] P. Lunghi, A. Tabilio, F. Lo-Coco, P. G. Pelicci and A. Bonati, "Arsenic Trioxide (ATO) and MEK1 Inhibition Synergize to Induce Apoptosis in Acute Promyelocytic Leukemia Cells,” Leukemia, Vol. 19, No. 2, 2005, pp. 234-244. doi:10.1038/sj.leu.2403585

[7] P. Lunghi, A. Costanzo, L. Salvatore, et al., "MEK1 Inhibition Sensitizes Primary Acute Myelogenous Leukemia to Arsenic Trioxide-Induced Apoptosis,” Blood, Vol. 107, No. 11, 2006, pp. 4549-4553. doi:10.1182/blood-2005-07-2829

[8] S. Takahashi, H. Harigae, H. Yokoyama, et al., "Synergistic Effect of Arsenic Trioxide and Flt3 Inhibition on Cells with Flt3 Internal Tandem Duplication,” International Journal of Hematology, Vol. 84, No. 3, 2006, pp. 256-261. doi:10.1532/IJH97.06076

[9] S. Takahashi, H. Harigae, J. Kameoka, T. Sasaki and M. Kaku, "AML1B Transcriptional Repressor Function is Impaired by the Flt3 Internal Tandem Duplication," British Journal of Haematology, Vol. 130, No. 3, 2005, pp. 428-436. doi:10.1186/1756-8722-4-13

[10] S. Takahashi, H. Harigae, M. Kaku, T. Sasaki and J. D. Licht, "Flt3 Mutation Activates p21(WAF1/CIP1) Gene Expression through the Action of STAT5,” Biochemical and Biophysical Research Communications, Vol. 316, No. 1, 2004, pp. 85-92. doi:10.1186/1756-8722-4-36

[11] M. Hirosawa, M. Nakahara, R. Otosaka, et al., "The p38 Pathway Inhibitor SB202190 Activates MEK/MAPK to Stimulate the Growth of Leukemia Cells,” Leukemia Research, Vol. 33, No. 5, 2009, pp. 693-699. doi:10.1016/j.leukres.2008.09.028

[12] J. Wen, H. Y. Cheng, Y. Feng, et al., "P38 MAPK Inhibition Enhancing ATO-Induced Cytotoxicity Against Multiple Myeloma Cells,” British Journal of Haematology, Vol. 140, No. 2, 2008, pp. 169-180. doi:10.1111/j.1365-2141.2007.06895.x

[13] A. Verma, M. Mohindru, D. K. Deb, et al., "Activation of Rac1 and the p38 Mitogen-Activated Protein Kinase Pathway in Response to Arsenic Trioxide," Journal of Biological Chemistry, Vol. 277, No. 47, 2002, pp. 4498844995. doi:10.1074/jbc.M207176200

[14] P. Lunghi, N. Giuliani, L. Mazzera, et al., “Targeting MEK/MAPK Signal Transduction Module Potentiates
ATO-Induced Apoptosis in Multiple Myeloma Cells through Multiple Signaling Pathways,” Blood, Vol. 112, No. 6, 2008, pp. 2450-2462. doi:10.1182/blood-2007-10-114348

[15] D. Douer and M. S. Tallman, "Arsenic Trioxide: New Clinical Experience with an Old Medication in Hematologic Malignancies,” Journal of Clinical Oncology, Vol. 23, No. 10, 2005, pp. 2396-2410. doi:10.1200/JCO.2005.10.217

[16] M. Cavigelli, W. W. Li, A. Lin, et al., "The Tumor Promoter Arsenite Stimulates AP-1 Activity by Inhibiting a JNK Phosphatase,” The European Molecular Biology Organization Journal, Vol. 15, No. 22, 1996, pp. 62696279.

[17] S. Ludwig, A. Hoffmeyer, M. Goebeler, et al., "The Stress Inducer Arsenite Activates Mitogen-Activated Protein Kinases Extracellular Signal-Regulated Kinases 1 and 2 via a MAPK Kinase 6/p38-Dependent Pathway," Journal of Biological Chemistry, Vol. 273, No. 4, 1998, pp. 1917-1922. doi:10.1074/jbc.273.4.1917

[18] C. Huang, W. Y. Ma, J. Li, A. Goranson and Z. Dong, "Requirement of Erk, but Not JNK, for Arsenite-Induced Cell Transformation,” Journal of Biological Chemistry, Vol. 274, No. 21, 1999, pp. 14595-14601. doi:10.1074/jbc.274.21.14595

[19] P. Baines, J. Fisher, L. Truran, et al., “The MEK Inhibitor, PD98059, Reduces Survival but Does Not Block Acute Myeloid Leukemia Blast Maturation in vitro,” European Journal of Haematology, Vol. 64, No. 4, 2000, pp. 211218. doi:10.1034/j.1600-0609.2000.90139.x

[20] P. Lunghi, A. Tabilio, P. P. Dall'Aglio, et al., "Downmodulation of ERK Activity Inhibits the Proliferation and Induces the Apoptosis of Primary Acute Myelogenous Leukemia Blasts,” Leukemia, Vol. 17, No. 9, 2003, pp. 1783-1793. doi:10.1038/sj.leu.2403032

[21] M. A. Morgan, O. Dolp and C. W. Reuter, "Cell-CycleDependent Activation of Mitogen-Activated Protein Kinase (MEK-1/2) in Myeloid Leukemia Cell Lines and Induction of Growth Inhibition and Apoptosis by Inhibitors of RAS Signaling,” Blood, Vol. 97, No. 6, 2001, pp. 1823-1834. doi:10.1182/blood.V97.6.1823

[22] S. Takahashi, "Inhibition of the MEK/MAPK Signal Transduction Pathway Strongly Impairs the Growth of Flt3-ITD Cells," American Journal of Hematology, Vol. 81, No. 2, 2006, pp. 154-155. doi:10.1002/ajh.20520 